\title{
The Ernst Equation as a Motion on a Universal Grassmann Manifold
}

\author{
K. Nagatomo \\ Department of Mathematics, Osaka University, Toyonaka, Osaka 560, Japan
}

\begin{abstract}
We find a linearization of the Ernst equation by means of universal Grassmann manifold (UGM) techniques. All local analytic solutions defined at the origin are obtained by solving an initial value problem for a linear differential equation on a UGM. We give an explicit formula which represents solutions of the Ernst equation. By using this formula, we generate several special solutions.
\end{abstract}

\section{Introduction}

The method of the universal Grassmann manifold (UGM) due to Sato $([1,2])$ provides us a unified framework of linearizing various kinds of soliton equations. Many soliton equations are equivalent to a system of linear differential equations on the UGM, which is completely integrable in the sense of Frobenius (Mulase [3]). Recently Takasaki [4] has shown that the equation of self-dual Yang-Mills fields, which is a typical example of the higher dimensional integrable system, is also linearized on the UGM. The $\infty \times \infty$ matrix $\xi$ introduced in [4], which represents a frame of a point of the UGM, has also been used with success in analyzing Witten's gauge field equations [5]. In this paper we investigate a linearization of the Ernst equation by using this $\infty \times \infty$ matrix. One of the immediate consequences of our main results is that all local analytic solutions defined at the origin are obtained by solving an initial value problem for a linear differential equation on the UGM.

The Ernst equation is a system of nonlinear differential equations for two unknown functions $f=f(z, \rho)$ and $e=e(z, \rho)([6])$ :

$$
\begin{aligned}
f \nabla^{2} f-\left(\partial_{z} f\right)^{2}-\left(\partial_{\rho} f\right)^{2}+\left(\partial_{z} e\right)^{2}+\left(\partial_{\rho} e\right)^{2} & =0, \\
f \nabla^{2} e-2\left(\partial_{z} f \cdot \partial_{z} e+\partial_{\rho} f \cdot \partial_{\rho} e\right) & =0
\end{aligned}
$$

in $\mathbf{R}_{z} \times \mathbf{R}_{\rho}^{+}$, where $\nabla^{2}=\partial_{\rho}^{2}+(1 / \rho) \partial_{\rho}+\partial_{z}^{2}$ and $\mathbf{R}_{\rho}^{+}=\{\rho \in \mathbf{R}: \rho>0\}$. In what follows we consider a formal power series solution at $(z, \rho)=(0,0)$, which is uniquely determined by its value at $\rho=0([7])$. We shall give a formula which represents formal power series solutions. It follows immediately from this formula that if the 
initial value at $\rho=0$ is analytic, the corresponding formal solution actually converges.

It has been known that the Ernst equation is the integrability condition of a system of linear differential equations: Hauser-Ernst [8], Belinsky-Zakharov[9], Maison[10], etc. The linear system used in our approach is given for a matrix form (Nakamura[11]) of the Ernst equation. Although our system coincides with that of Belinsky-Zakharov after an appropriate change of the spectral parameter, we impose on the solutions of our linear system, called the wave function, a different supplementary condition. Our linear system has then a unique wave function which is analytic at $(z, \rho, \lambda)=(0,0, \infty)$, where $\lambda$ stands for the spectral parameter, a fact which is one of the reasons why we use this linear system. Our basic result is that this unique wave function is obtained by solving an initial value problem with an initial value at $\rho=0$ for one of the two equations of this system. The other equation can be coded into a condition on the initial value. The linear differential equation to be solved here plays a similar role as Sato equation for Kadomtsev-Petriashvili equation.

Once the problem of finding a wave function is reduced to an initial value problem for a single linear differential equation, we can use the UGM techniques to solve the Ernst equation. In general our formula representing the solutions of the Ernst equation involves manipulations of the $\infty \times \infty$ matrices, but if the initial value satisfies a certain condition, all calculations are done by finite dimensional linear algebra. As examples, we construct several rational solutions by our method.

It is worthwhile noting that the Ernst equation is an analytic elliptic system, and hence any $C^{3}$ solution at $(z, \rho)=(0,0)$ turns out to be analytic ([8]). Indeed, there exists a solution which has a singularity on the axis $\rho=0$. A typical example of such solutions is the determinantal solution which has a pole singularity on the axis $\rho=0$ (Kyriakopoulos[13], Vein[14], Candler and Freeman[15], Nagatomo[16]). Unfortunately we cannot analyze such solutions, because the initial value problem with an initial value at $\rho=0$ discussed in this paper is irrelevant to these solutions.

In the next section we state our main results and give the organization of this paper.

\section{Statement of Results}

Let $\mathscr{C}$ be the set of all $2 \times 2$ matrices with components in $\mathbf{R}$ (real numbers). Throughout this paper $\mathscr{C} \llbracket z \rrbracket$ and $\mathscr{C} \llbracket z, \rho \rrbracket$ denote respectively the set of all formal power series in $z$ and $(z, \rho)$ with coefficients in $\mathscr{C}$.

Following Nakamura[11] we rewrite the Ernst equation in matrix form. We assume $f \neq 0$, for the function $f$ represents physically a non-vanishing coefficient of a Lorentz metric. Define a square matrix $\tau(z, \rho)$ by

$$
\tau(z, \rho)=\left[\begin{array}{cc}
\frac{f^{2}+e^{2}}{f} & e \\
\frac{e}{f} & -\frac{1}{f}
\end{array}\right] .
$$


Then the Ernst equation is expressed as

$$
\begin{gathered}
\partial_{z}\left(\rho \partial_{z} \tau \cdot \tau^{-1}\right)+\partial_{\rho}\left(\rho \partial_{\rho} \tau \cdot \tau^{-1}\right)=0, \\
\operatorname{det} \tau=1, \quad{ }^{t} \tau=\tau .
\end{gathered}
$$

We call Eq. (1.2) the matrix Ernst equation.

Let us consider the following initial value problem for Eq. (1.2) with the following condition on the initial value:

IVP-1

$$
\begin{cases}\partial_{z}\left(\rho \partial_{z} \tau \cdot \tau^{-1}\right)+\partial_{\rho}\left(\rho \partial_{\rho} \tau \cdot \tau^{-1}\right)=0, & \tau(z, \rho) \in \mathscr{C} \llbracket z, \rho \rrbracket, \\ \left.\tau(z, \rho)\right|_{\rho=0}=\tau(z), & \tau(z) \in \mathscr{C} \llbracket z \rrbracket^{\times},\end{cases}
$$

where $\mathscr{C} \llbracket\left[z \rrbracket^{\times}\right.$denotes the set of invertible elements of $\mathscr{C} \llbracket z \rrbracket$. We shall prove that this initial value problem is uniquely solvable (Proposition 2.1).

In [7] we have shown the unique solvability of the initial value problem for the Ernst equation with the initial value $\left.f(z, \rho)\right|_{\rho=0}=f(z) \in \mathbf{R} \llbracket z \rrbracket^{\times},\left.e(z, \rho)\right|_{\rho=0}=$ $e(z) \in \mathbf{R} \llbracket z]$. By virtue of this fact, we obtain the result that if the initial data satisfies condition (1.3), then the corresponding solution of IVP-1 also satisfies this (Corollary 2.3), so that we may restrict ourselves to analyzing Eq. (1.2).

To introduce a linear system associated with Eq. (1.2), we rewrite first Eq. (1.2) by using the matrices $P=\partial_{z} \tau \cdot \tau^{-1}$ and $Q=\partial_{\rho} \tau \cdot \tau^{-1}$ as

$$
\begin{aligned}
\partial_{z}(\rho P)+\partial_{\rho}(\rho Q) & =0, \\
\partial_{\rho} P-\partial_{z} Q+[P, Q] & =0 .
\end{aligned}
$$

Consider then the following system of linear differential equations for the unknown function $W=W(z, \rho, \lambda)$,

$$
\begin{aligned}
& D_{1} W=P W, \\
& D_{2} W=Q W,
\end{aligned}
$$

where $D_{1}=\partial_{z}-\lambda \rho \partial_{\rho}+2 \lambda^{2} \partial_{\lambda}$ and $D_{2}=\lambda \rho \partial_{z}+\partial_{\rho}$. It is clear that Eqs. (1.5) are involutive if and only if $P$ and $Q$ satisfy Eqs. (1.4).

Let $\tau(z, \rho)$ be a solution of Eq. (1.2) with an initial value $\left.\tau(z, \rho)\right|_{\rho=0}=\tau(z)$, $\tau(z) \in \mathscr{C} \llbracket z \rrbracket$. Then Eqs. (1.5) have a unique solution which takes the form $W(z, \rho, \hat{\lambda})=$ $1_{2}+\sum_{j=1}^{\infty} w_{l}(z, \rho) \lambda^{-j}$, where $w_{\jmath}(z, \rho) \in \mathscr{C} \llbracket z, \rho \rrbracket$ and $1_{2}$ stands for $2 \times 2$ unit matrix (Proposition 3.1). Further the value of $W(z, \rho, \lambda)$ at $\rho=0$ is given by

$$
\left.W(z, \rho, \lambda)\right|_{\rho=0}=\tau(z) \cdot\left[\tau\left(z+\frac{1}{2 \lambda}\right)\right]^{-1} \cdot \quad \text { (Proposition 3.3) }
$$

This result cnables us to study Eqs. (1.5) by using an initial value problem with an initial value at $\rho=0$. In fact, the system of Eqs. (1.5) is equivalent to the following initial value problem for $W(z, \rho, \lambda)=1_{2}+\sum_{j=1}^{\infty} w_{j}(z, \rho) \hat{\lambda}^{-j}, w_{j}(z, \rho) \in \mathscr{C} \llbracket z, \rho \rrbracket$ (Proposition 3.5): 
IVP-2

$$
\left\{\begin{array}{l}
D_{2} W=Q W \\
\left.W(z, \rho, \lambda)\right|_{\rho=0}=\tau(z) \cdot[\tau(z+1 / 2 \lambda)]^{-1}
\end{array}\right.
$$

Obviously, every wave function has the following property:

(P) $D_{1} W \cdot W^{-1}$ and $D_{2} W \cdot W^{-1}$ are independent of the variable $\lambda$.

It is important to notice that the above property $(\mathrm{P})$ completely characterizes the wave functions. Namely, if a formal power series $W(z, \rho, \lambda)=1_{2}+\sum_{j=1}^{\infty} w_{j}(z, \rho) \lambda^{-j}$, $w_{j}(z, \rho) \in \mathscr{C}[z, \rho]$ has the property $(\mathrm{P})$, then $P=D_{1} W \cdot W^{-1}$ and $Q=D_{2} W \cdot W^{-1}$, which are functions only of $z$ and $\rho$, satisfy Eqs. (1.4) (Lemma 4.1). satisfies

One of our main results is that if $W(z, \rho, \lambda)=1_{2}+\sum_{j=1}^{\infty} w_{j}(z, \rho) \lambda^{-j}, w_{j}(z, \rho) \in \mathscr{C} \llbracket z, \rho \rrbracket$

$$
\left\{\begin{array}{l}
D_{2} W \cdot W^{-1} \text { is independent of the variable } \lambda, \\
\left.W(z, \rho, \lambda)\right|_{\rho=0}=\tau(z) \cdot[\tau(z+1 / 2 \lambda)]^{-1},
\end{array}\right.
$$

then $D_{1} W \cdot W^{-1}$ is also independent of the variable $\lambda$. Furthermore every solution $\tau(z, \rho)$ of Eq. (1.2) can be obtained from $W$ with a suitably chosen initial value $\left.\tau(z, \rho)\right|_{\rho=0}=\tau(z)$ (Theorem 4.3, Remark 4.4).

The formal power series which satisfies condition (1.7) is obtained by solving an initial value problem for a linear differential equation on UGM. The first statement of (1.7) is equivalent to the following infinite series of differential equations for $w_{j}(z, \rho) \in \mathscr{C} \llbracket z, \rho \rrbracket$ :

$$
\rho \partial_{z} w_{j}+\partial_{\rho} w_{j-1}=\rho \partial_{z} w_{1} \cdot w_{j-1} \text { for any } j \in \mathbf{N} .
$$

A similar equation has already appeared in the study of the self-dual Yang-Mills (SDYM) equation (Takasaki[4]). In terms of an $\infty \times \infty$ matrix $\zeta=\left(\check{\zeta}_{i j}\right)_{l \in \mathbf{Z}, j<0}$ introduced in [4] (see Sect. V for the definition of $\xi$ ) Eqs. (1.8) are expressed as

$$
\rho \partial_{z} \xi_{i+1, j}+\partial_{\rho} \xi_{i j}-\xi_{i,-1} \rho \hat{o}_{z} \xi_{0 j}=0 .
$$

The second condition of (1.7) determines the initial value of $\xi$ at $\rho=0$. As in Takasaki[4], we can generate the solution of Eqs. (1.9) with the specified initial value from a solution $\widetilde{\xi}$ of the linear equation

$$
\rho \partial_{z} \tilde{\xi}_{i+1, j}+\partial_{\rho} \tilde{\xi}_{i j}=0 \text { for } i \in \mathbf{Z}, j<0
$$

(Theorem 5.2).

To generate special solutions of the Ernst equation, we consider the case when the initial data $\tau(z)$ be a polynomial of $z$ such that $\operatorname{det} \tau(z)=1$. Then the matrix $\xi$ is reduced to a finite size one and therefore by using finite dimensional linear algebra we can construct solutions of Eqs. (1.9), which are rational functions of both $z$ and $\rho$ (Corollary 5.4). We shall give several special solutions of the Ernst equation with such initial values.

The present paper is organized as follows. In Sect. II the initial value problem 
IVP-1 is considered and its unique solvability is proved. We review the inverse scattering formalism for the Ernst equation in Sect. III, where we prove that every wave function is uniquely determined by the initial value problem IVP-2. Our main result (Theorem 4.3) is stated and proved in Sect. IV and the Ernst equation is linearized by the UGM method in Sect. V, where several simple examples are also discussed.

\section{Initial Value Problem IVP-1}

Let us consider the following initial value problem with an initial value at $\rho=0$ : IVP-1:

$$
\begin{cases}\partial_{z}\left(\rho \partial_{z} \tau \cdot \tau^{-1}\right)+\partial_{\rho}\left(\rho \partial_{\rho} \tau \cdot \tau^{-1}\right)=0, & \tau(z, \rho) \in \mathscr{C} \llbracket z, \rho \rrbracket, \\ \left.\tau(z, \rho)\right|_{\rho=0}=\tau(z), & \tau(z) \in \mathscr{C} \llbracket z \rrbracket^{\times} .\end{cases}
$$

This initial value problem is uniquely solvable.

Proposition 2.1. The initial value problem IVP-1 has a unique solution.

Proof. Suppose given a solution $\tau(z, \rho)$ of IVP-1. We first show that $\left.\partial_{\rho}^{k} \tau(z, \rho)\right|_{\rho=0}$ is uniquely determined inductively from the initial value $\tau(z)$. Differentiating $k$ times both sides of the matrix Ernst equation:

$$
\partial_{\rho} \tau=-\rho \partial_{\rho}\left(\rho \partial_{\rho} \cdot \tau^{-1}\right) \cdot \tau-\rho \partial_{z}\left(\partial_{z} \tau \cdot \tau^{-1}\right) \cdot \tau,
$$

we have

$$
\begin{aligned}
\partial_{\rho}^{k+1} \tau= & -\rho \partial_{\rho}^{k}\left(\partial_{\rho}\left(\partial_{\rho} \tau \cdot \tau^{-1}\right) \cdot \tau\right)-k \partial_{\rho}^{k-1}\left(\partial_{\rho}\left(\partial_{\rho} \tau \cdot \tau^{-1}\right) \cdot \tau\right) \\
& -\rho \partial_{\rho}^{k}\left(\partial_{z}\left(\partial_{z} \tau \cdot \tau^{-1}\right) \cdot \tau\right)-k \partial_{\rho}^{k-1}\left(\partial_{z}\left(\partial_{z} \tau \cdot \tau^{-1}\right) \cdot \tau\right) .
\end{aligned}
$$

Substitute the identity

$$
\begin{aligned}
\partial_{\rho}^{k-1}\left(\partial_{\rho}\left(\partial_{\rho} \tau \cdot \tau^{-1}\right) \cdot \tau\right) & =\partial_{\rho}^{k-1}\left(\partial_{\rho}^{2} \tau-\partial_{\rho} \tau \cdot \tau^{-1} \partial_{\rho} \tau\right) \\
& =\partial_{\rho}^{k+1} \tau-\partial_{\rho}^{k-1}\left(\partial_{\rho} \tau \cdot \tau^{-1} \partial_{\rho} \tau\right)
\end{aligned}
$$

into Eq. (2.1) and set $\rho=0$. Then

$$
\begin{aligned}
\left.\partial_{\rho}^{k+1} \tau(z, \rho)\right|_{\rho=0}= & -\left.k \partial_{\rho}^{k+1} \tau(z, \rho)\right|_{\rho=0}+\left.k \partial_{\rho}^{k-1}\left(\partial_{\rho} \tau \cdot \tau^{-1} \partial_{\rho} \tau\right)(z, \rho)\right|_{\rho=0} \\
& -\left.k \partial_{\rho}^{k-1}\left(\partial_{z}\left(\partial_{z} \tau \cdot \tau^{-1}\right) \cdot \tau\right)(z, \rho)\right|_{\rho=0},
\end{aligned}
$$

and hence

$$
\begin{aligned}
\left.\frac{k+1}{k} \partial_{\rho}^{k+1} \tau(z, \rho)\right|_{\rho=0}= & \left.\partial_{\rho}^{k-1}\left(\partial_{\rho} \tau \cdot \tau^{-1} \partial_{\rho} \tau\right)(z, \rho)\right|_{\rho=0} \\
& -\left.\partial_{\rho}^{k-1}\left(\partial_{z}\left(\partial_{z} \tau \cdot \tau^{-1}\right) \cdot \tau\right)(z, \rho)\right|_{\rho=0} .
\end{aligned}
$$

Clearly, the right-hand side of the above equation is a polynomial of $\partial_{z}^{l} \tau_{j}(z)$ $(i=0,1,2, j=0, \ldots, k)$ and $\tau_{0}(z)^{-1}$, where $\tau_{j}(z)=\left.\partial_{\rho}^{j} \tau(z, \rho)\right|_{\rho=0}$. Therefore, $\tau_{k}(z), k \in \mathbf{N}$ is uniquely determined from $\tau_{0}(z)=\tau(z)$.

Let $H_{k}\left(\tau_{0}^{-1}, \tau_{j}, \partial_{z} \tau_{j}, \partial_{z}^{2} \tau_{j}\right)$ denote the right-hand side of Eq. (2.2). Define $\tau_{k+1}$ 
inductively by

$$
\tau_{k+1}(z)=\frac{k}{k+1} H_{k}\left(\tau_{0}^{-1}, \tau_{j}, \partial_{z} \tau_{j}, \partial_{z}^{2} \tau_{j}\right)(z), \quad \tau_{0}(z)=\tau(z) .
$$

Then $\tau(z, \rho)=\sum_{k=0}^{\infty}\left(\tau_{k}(z) / k !\right) \rho^{k}$ is a unique solution of IVP -1 .

Remark 2.2. Let $\tau(z, \rho)=\sum_{k=0}^{\infty}\left(\tau_{k}(z) / k !\right) \rho^{k}$ be a solution of IVP-1. Since $\tau(z,-\rho)$ is also a solution of the matrix Ernst equation with the same initial value as $\tau(z, \rho)$, we have $\tau(z, \rho)=\tau(z,-\rho)$ from Proposition 2.1. Hence $\tau_{2 k-1}(z)=0$ for $k \in \mathbf{N}$, i.e., $\tau(z, \rho)$ is an even function of $\rho$.

To generate a solution of the original Ernst equation we have to find a solution $\tau(z, \rho)$ of IVP-1 such that $\operatorname{det} \tau=1$ and ${ }^{t} \tau=\tau$.

Corollary 2.3. If an initial data $\tau(z) \in \mathscr{C} \llbracket z \rrbracket^{\times}$satisfies the supplementary conditions $\operatorname{det} \tau(z)=1$ and ${ }^{t} \tau(z)=\tau(z)$, then the unique solution of IVP-1 also satisfies $\operatorname{det} \tau(z, \rho)=$ 1 and ${ }^{t} \tau(z, \rho)=\tau(z, \rho)$.

Proof. Define $f(z) \in \mathscr{C}[z]^{\times}$and $e(z) \in \mathscr{C} \llbracket z \rrbracket$ by

$$
\tau(z)=\left[\begin{array}{cc}
\frac{f^{2}+e^{2}}{f} & e \\
e & -\frac{1}{f}
\end{array}\right] .
$$

Then by Proposition 2.1 in [7] there exists a solution $f(z, \rho), e(z, \rho)$ of the Ernst equation such that $\left.f(z, \rho)\right|_{\rho=0}=f(z)$ and $\left.e(z, \rho)\right|_{\rho=0}=e(z)$. Define $\tau(z, \rho)$ by (1.1). Then $\tau(z, \rho)$ is a unique solution of IVP-1 and satisfies $\operatorname{det} \tau(z, \rho)=1$ and ${ }^{t} \tau(z, \rho)=\tau(z, \rho)$.

It follows from Corollary 2.2 that the solution space of the Ernst equation is specified by the initial values of IVP-1, so that we may only consider IVP-1.

\section{Initial Value Problem for Wave Functions}

As mentioned in Sect. 1, the matrix Ernst equation is expressed as the integrability condition of a system of linear differential equations. A solution of this system is called a wave function. We are interested in a wave function which is "analytic" at $(z, \rho, \lambda)=(0,0, \infty)$. Such a wave function surely exists uniquely.

Proposition 3.1. Let $\tau(z, \rho)$ be a solution of the matrix Ernst equation. Then there exists a unique $W(z, \rho, \lambda)=1_{2}+\sum_{j=1}^{\infty} w_{j}(z, \rho) \lambda^{-j}, w_{j}(z, \rho) \in \mathscr{C} \llbracket z, \rho \rrbracket$ such that

$$
D_{1} W=P W, \quad D_{2} W=Q W,\left.\quad W(z, \rho, \lambda)\right|_{\lambda=0}=1_{2} .
$$

Proof. Substituting $W(z, \rho, \lambda)=1_{2}+\sum_{j=1}^{\infty} w_{j}(z, \rho) \lambda^{-j}, w_{j}(z, \rho) \in \mathscr{C} \llbracket z, \rho \rrbracket$ into Eqs. (3.1), 
we have an infinite series of differential equations for $w_{j}(z, \rho), j \in \mathbf{N}$ :

$$
\begin{gathered}
\rho \partial_{\rho} w_{j}(z, \rho)+2 j w_{j}(z, \rho)=\hat{c}_{z} w_{j-1}(z, \rho)-P w_{j-1}(z, \rho), \\
\rho \partial_{z} w_{j}(z, \rho)=-\partial_{\rho} w_{j-1}(z, \rho)+Q w_{j-1}(z, \rho),
\end{gathered}
$$

where we put $w_{0}=1_{2}$. We use induction to show the existence of $w_{j}(z, \rho) \in \mathscr{C} \llbracket z, \rho \rrbracket$ subject to (3.2-j) and (3.3-j).

We can easily find a special solution of Eqs. (3.2.-1) and (3.3-1), which is given by

$$
w_{1}(z, \rho)=-\frac{1}{2} P(0, \rho)+\int_{0}^{\rho} \frac{1}{2}\left(\frac{t}{\rho}\right)^{2} \partial_{t} P(0, t) d t+\int_{0}^{z} \frac{1}{\rho} Q(s, \rho) d s .
$$

Because $Q(z, \rho)=\partial_{\rho} \tau \cdot \tau^{-1}$ is an odd function of $\rho$ (see Remark 2.2), we observe that $Q(z, \rho) / \rho$, and therefore $w_{1}(z, \rho)$, is an element of $\mathscr{E} \llbracket z, \rho \rrbracket$. Further it follows from the above expression that $w_{1}(z, \rho)$ is an even function of $\rho$.

Assume that there exists a solution $w_{j}(z, \rho) \in \mathscr{C} \llbracket z, \rho \rrbracket$ of Eqs. (3.2-j) and (3.3-j), which is an even function of $\rho$. Let us define $w_{j+1}(z, \rho)$ by

$$
\begin{aligned}
w_{j+1}(z, \rho)= & \frac{1}{2(j+1)} \int_{0}^{\rho} \rho^{-2(j+1)} s^{2 j+1}\left\{\partial_{z} w_{j}(0, s)-P(0, s) w_{j}(0, s)\right\} d s \\
& -\int_{0}^{z} \frac{1}{\rho}\left\{\partial_{\rho} w_{j}(s, \rho)-Q(s, \rho) w_{j}(s, \rho)\right\} d s .
\end{aligned}
$$

We can easily verify that $w_{j+1}$ satisfies Eqs. $(3.2-\mathrm{j}+1)$ and $(3.2-\mathrm{j}+1)$. We have $w_{j+1}(z, \rho) \in \mathscr{C} \llbracket z, \rho \rrbracket$, because $\partial_{\rho} w_{J}(z, \rho)$ and $Q(z, \rho)$ are odd functions of $\rho$. Further it follows from (3.4) that $w_{j+1}(z, \rho)$ is an even function of $\rho$. Hence by induction we conclude the existence of $w_{j}(z, \rho)$. $j \in \mathbf{N}$ subject to Eqs. (3.2) and (3.3).

We can prove the uniqueness of the wave function $W$ as follows. Let $W$ and $\hat{W}$ be solutions of Eqs. (3.1). Then $u$ defined by

$$
u=W^{-1} \cdot \hat{W}
$$

satisfies $D_{1} u=D_{2} u=0$. In the following Lemma we show $u=1_{2}$, so that we have $W=\hat{W}$.

Lemma 3.2. If $u(z, \rho, \hat{\lambda})=1_{2}+\sum_{j=1}^{\infty} u_{j}(z, \rho) \lambda^{-j}, u_{j}(z, \rho) \in \mathscr{C} \llbracket z, \rho \rrbracket$ satisfies $D_{1} u=D_{2} u=0$, then $u(z, \rho, \lambda)=1_{2}$.

Proof. We first note that the functions $u_{j}(z, \rho), j \in \mathbf{N}$ satisfy

$$
\begin{gathered}
\rho \partial_{\rho} u_{j}+2 j u_{j}=\partial_{z} u_{j-1}, \\
\rho \partial_{z} u_{j}=-\partial_{\rho} u_{j-1} .
\end{gathered}
$$

We have $\rho \partial_{\rho} u_{1}+2 u_{1}=0$ and $\rho \partial_{z} u_{1}=0$ when $j=1$. With the condition $u_{1}(z, \rho) \in$ $\mathscr{C} \llbracket z, \rho \rrbracket$, these equations yield $u_{1}(z, \rho)=0$. Assume now that $u_{j}(z, \rho)=0$. Then similarly as above we can show $u_{j+1}(z, \rho)=0$. Hence induction on $j$ proves $u(z, \rho, \lambda)=1_{2}$.

We have seen that any solution of the matrix Ernst equation is uniquely determined by its value at $\rho=0$ (Proposition 2.1). We can represent the value of the unique solution of Eqs. (3.1) at $\rho=0$ in terms of the initial value $\tau(z)$. 
Proposition 3.3. Let $W(z, \rho, \lambda)=1_{2}+\sum_{j=1}^{\infty} w_{j}(z, \rho) \lambda^{-j}, w_{j}(z, \rho) \in \mathscr{C} \llbracket z, \rho \rrbracket$ be the unique solution of Eqs. (3.1). Then

$$
\left.W(z, \rho, \lambda)\right|_{\rho=0}=\tau(z) \cdot\left[\tau\left(z+\frac{1}{2 \lambda}\right)\right]^{-1} .
$$

Proof. By setting $\rho=0$ in Eqs. (1.5a):

$$
\left(\partial_{z}-\lambda \rho \partial_{\rho}+2 \lambda^{2} \partial_{\lambda}\right) W(z, \rho, \lambda)=\partial_{z} \tau \cdot \tau^{-1} W(z, \rho, \lambda),
$$

we find

$$
\left(\partial_{z}+2 \lambda^{2} \partial_{\lambda}\right) W(z, 0, \lambda)=\partial_{z} \tau(z) \cdot \tau(z)^{-1} \cdot W(z, 0, \lambda)
$$

In terms of $w_{j}$, this takes the form

$$
2 j w_{j}(z, 0)=\partial_{z} w_{j-1}(z, 0)-P(z, 0) w_{j-1}(z, 0), \text { for } j \in \mathbf{N} .
$$

Thus $w_{j}(z, 0)$ is uniquely determined by Eq. (3.6). On the other hand, the function $\tau(z) \cdot[\tau(z+1 / 2 \lambda)]^{-1}$ clearly satisfies Eqs. (3.6). Hence we have proved the proposition.

Remark 3.4. Equation (1.5b) gives no influence on the form of $W(z, 0, \lambda)$, because when $\rho=0$, we have $D_{2} W=0$ and $Q=0$ by virtue of the fact that $W$ is even and $Q$ is odd with respect to the variable $\rho$.

Let $W$ be the unique solution of Eqs. (3.1). Then it follows from Proposition 3.3 that $W$ is a solution of the initial value problem

IVP-2

$$
\begin{aligned}
& D_{2} W=Q W, \quad W(z, \rho, \lambda)=1_{2}+\sum_{j=1}^{\infty} w_{j}(z, \rho) \lambda^{-j}, \quad w_{j}(z, \rho) \in \mathscr{C} \llbracket[z, \rho \rrbracket, \\
& \left.W(z, \rho, \lambda)\right|_{\rho=0}=\tau(z) \cdot[\tau(z+1 / 2 \lambda)]^{-1}, \quad \tau(z) \in \mathscr{C} \llbracket z \rrbracket^{\times} .
\end{aligned}
$$

Conversely every solution of IVP-2 satisfies Eqs. (3.1), whence the initial value problem IVP-2 is equivalent to Eqs. (3.1).

We first note that a solution of IVP-2 is unique, because IVP-2 is written by $w_{j}(z, \rho)$ as

$$
\rho \partial_{z} w_{j}=-\partial_{\rho} w_{j-1}+Q w_{j-1} .
$$

Suppose now given a solution $W$ of IVP-2. We have to prove $D_{1} W=P W$. Let $\hat{W}$ be a solution of Eqs. (3.1), so that $\hat{W}$ satisfies IVP-2. Then the uniqueness of IVP-2 yields $W=\hat{W}$, a fact which proves $D_{1} W=P W$.

Thus we have proved the following proposition.

Proposition 3.5. Equations (3.1) is equivalent to IVP-2.

\section{Main Theorem}

Let $\tau(z, \rho)$ be a solution of the matrix Ernst equation with an initial value $\left.\tau(z, \rho)\right|_{\rho=0}=\tau(z)$ and let $W$ be the unique solution of Eqs. (3.1). It follows from 
Proposition 3.3 that $W$ has the following property:

$$
\left\{\begin{array}{l}
D_{2} W \cdot W^{-1} \text { is independent of the variables } \lambda, \\
\left.W(z, \rho, \lambda)\right|_{\rho=0}=\tau(z) \cdot[\tau(z+1 / 2 \lambda)]^{-1} .
\end{array}\right.
$$

In the following we shall show that the above property completely characterizes a wave function. Namely, we shall prove that if a formal power series $W(z, \rho, \lambda)=$ $1_{2}+\sum_{j=1}^{\infty} w_{j}(z, \rho) \lambda^{-j}, w_{j}(z, \rho) \in \mathscr{C} \llbracket z, \rho \rrbracket$ has the above property, then $D_{1} W \cdot W^{-1}$ is independent of the variable $\lambda$.

The solution of the matrix Ernst equation with the initial value $\left.\tau(z, \rho)\right|_{\rho=0}=\tau(z)$ can be generated using such $W$ as follows.

Lemma 4.1. Let $W(z, \rho, \lambda)=1_{2}+\sum_{j=1}^{\infty} w_{j}(z, \rho) \lambda^{-j}, w_{j}(z, \rho) \in \mathscr{C} \llbracket z, \rho \rrbracket$ be a formal power series such that $D_{1} W \cdot W^{-1}$ and $D_{2} W \cdot W^{-1}$ are independent of the variable $\lambda$. Then

(1) $P(z, \rho)=D_{1} W \cdot W^{-1}$ and $Q(z, \rho)=D_{2} W \cdot W^{-1}$ satisfy Eqs. (1.4).

(2) The linear differential equations

$$
\hat{\partial}_{z} \tau=P \tau, \quad \partial_{\rho} \tau=Q \tau
$$

have a unique solution up to a multiplicative factor.

(3) Any solution $\tau(z, \rho)$ of Eqs. (4.1) satisfies the matrix Ernst equation.

Proof. A simple calculation shows that

$$
\left[D_{1}, D_{2}\right] W=\left(D_{1} Q-D_{2} P+[Q, P]\right) W .
$$

By virtue of the commutator relation $\left[D_{1}, D_{2}\right]=\lambda D_{2}$, we get

$$
\lambda Q W=\left(D_{1} Q-D_{2} P+[Q, P]\right) W,
$$

so that

$$
\lambda Q=D_{1} Q-D_{2} P+[Q, P],
$$

where we use the invertibility of $W$. This proves the first statement. The second and third statements are immediately shown by Eqs. (1.4).

Lemma 4.2. Let $W(z, \lambda)$ be a formal power series of the form

$$
W(z, \lambda)=1_{2}+\sum_{j=1}^{\infty} w_{j}(z) \lambda^{-j}, \quad w_{j}(z) \in \mathscr{C} \llbracket z \rrbracket .
$$

Then there exists a unique

$$
W(z, \rho, \lambda)=1_{2}+\sum_{j=1}^{\infty} w_{j}(z, \rho) \lambda^{-\jmath}, \quad w_{j}(z, \rho) \in \mathscr{C} \llbracket z, \rho \rrbracket
$$

such that $\left.W(z, \rho, \lambda)\right|_{\rho=0}=W(z, \lambda)$ and that $D_{2} W \cdot W^{-1}$ is independent of the variable $\lambda$. Proof. If $\left(\lambda \rho \partial_{z}+\partial_{\rho}\right) W(z, \rho, \lambda) \cdot[W(z, \rho, \lambda)]^{-1}$ is independent of the variable $\lambda$, we find

$$
\left(\lambda \rho \partial_{z}+\partial_{\rho}\right) W(z, \rho, \lambda)=\rho \partial_{z} w_{1}(z, \rho) \cdot W(z, \rho, \lambda) .
$$


Substituting the expression $W(z, \rho, \lambda)=1_{2}+\sum_{j=1}^{\infty} w_{j}(z, \rho) \lambda^{-j}$ into the above, we get

$$
\partial_{\rho} w_{j}(z, \rho)=-\rho \partial_{z} w_{j+1}(z, \rho)+\rho \partial_{z} w_{1}(z, \rho) \cdot w_{j}(z, \rho) .
$$

Hence we first see

$$
\left.\partial_{\rho} w_{j}(z, \rho)\right|_{\rho=0}=0 \text { for } j=0,1,2, \ldots .
$$

By differentiating $k$ times both sides of Eq. (4.2), we have

$$
\begin{aligned}
\partial_{\rho}^{k+1} w_{j}(z, \rho)= & -\rho \partial_{z} \partial_{\rho}^{k} w_{j+1}(z, \rho)-k \partial_{z} \partial_{\rho}^{k-1} w_{j+1}(z, \rho) \\
& +\sum_{r=0}^{k}\left(\begin{array}{l}
k \\
r
\end{array}\right)\left\{\rho \partial_{z} \partial_{\rho}^{r} w_{1}(z, \rho)+r \partial_{\rho}^{r-1} w_{1}(z, \rho)\right\} \cdot \partial_{\rho}^{k-r_{1}} w_{j}(z, \rho) .
\end{aligned}
$$

Then by setting $\rho=0$ we find

$$
\begin{aligned}
\left.\partial_{\rho}^{k+1} w_{j}(z, \rho)\right|_{\rho=0}= & -\left.k \partial_{z} \partial_{\rho}^{k-1} w_{j+1}(z, \rho)\right|_{\rho=0} \\
& +\left.\sum_{r=0}^{k} r\left(\begin{array}{l}
k \\
r
\end{array}\right) \partial_{z} \partial_{\rho}^{r-1} w_{1}(z, \rho) \partial_{\rho}^{k-r} w_{j}(z, \rho)\right|_{\rho=0} .
\end{aligned}
$$

Therefore $\left.\partial_{\rho}^{k+1} w_{j}(z, \rho)\right|_{\rho=0}, j \in \mathbf{N}$ is uniquely determined by $\left.\partial_{\rho}^{r} w_{j}(z, \rho)\right|_{\rho=0}, j \in \mathbf{N}$, $r \leqq k$. Thus by the induction $\left.\partial_{\rho}^{r} w_{J}(z, \rho)\right|_{\rho=0}$ is uniquely determined by the initial data $w_{j}(z), j \in \mathbf{N}$. This proves the lemma.

Theorem 4.3. Let $\tau(z) \in \mathscr{C}\left[z \rrbracket^{\times}\right.$. If a formal power series

$$
W(z, \rho, \lambda)=1_{2}+\sum_{j=1}^{\infty} w_{j}(z, \rho) \lambda^{-j}, \quad w_{j}(z, \rho) \in \mathscr{C} \llbracket z, \rho \rrbracket
$$

satisfies the conditions that

(1) $D_{2} W \cdot W^{-1}$ is independent of the variable $\lambda$,

(2) $\left.W(z, \rho, \lambda)\right|_{\rho=0}=\tau(z) \cdot[\tau(z+1 / 2 \lambda)]^{-1}$,

then $D_{1} W \cdot W^{-1}$ is also independent of the variable $\lambda$. Furthermore a unique solution $\tau(z, \rho)$ of the initial value problem

$$
\partial_{\rho} \tau(z, \rho)=Q(z, \rho) \cdot \tau(z, \rho),\left.\quad \tau(z, \rho)\right|_{\rho=0}=\tau(z),
$$

where $Q(z, \rho)=\rho \partial_{z} w_{1}(z, \rho)$, satisfies the matrix Ernst equation.

Proof. Let $\hat{\tau}(z, \rho)$ be a unique solution of the matrix Ernst equation such that

$$
\left.\hat{\tau}(z, \rho)\right|_{\rho=0}=\tau(z) .
$$

Let $\hat{W}(z, \rho, \lambda)=1_{2}+\sum_{j=1}^{\infty} \hat{w}_{j}(z, \rho) \lambda^{-j}$ be the wave function associated with $\hat{\tau}(z, \rho)$, i.e. a unique solution of the equation

$$
D_{1} \hat{W}=\hat{P} \hat{W}, \quad D_{2} \hat{W}=\hat{Q} \hat{W},
$$

where $\hat{P}=\partial_{z} \hat{\tau} \cdot \hat{\tau}^{-1}$ and $\hat{Q}=\partial_{\rho} \hat{\tau} \cdot \hat{\tau}^{-1}$. Then from Proposition 3.3, $\left.\hat{W}(z, \rho, \lambda)\right|_{\rho=0}=$ $\tau(z) \cdot[\tau(z+1 / 2 \lambda)]^{-1}$. Since $W$ and $\hat{W}$ satisfy the same initial condition, we have 
$W=\hat{W}$ by using Lemma 4.2. Hence $D_{1} W \cdot W^{-1}=D_{2} \hat{W} \cdot \hat{W}^{-1}=\hat{Q}$ is independent of the variable $\lambda$. This proves the first statement.

To prove the second statement, we first note $D_{2} W \cdot W^{-1}=\rho \partial_{z} w_{1}$. Because $Q$ and $P=D_{1} W \cdot W^{-1}$ give a solution of Eqs. (1.4), there exists a solution $\hat{\tau}(z, \rho)$ of the equation

$$
\partial_{z} \hat{\tau}=P \hat{\tau}, \quad \partial_{\rho} \hat{\tau}=Q \hat{\tau}
$$

which is unique up to a multiplicative factor. Indeed, $\hat{\tau}(z, \rho)$ is a solution of the matrix Ernst equation. Clearly, $\left.P\right|_{\rho=0}=\partial_{z} \tau(z) \cdot[\tau(z)]^{-1}$. Hence by using the first of Eqs. (4.4) at $\rho=0$ we may assume $\left.\hat{\tau}(z, \rho)\right|_{\rho=0}=\tau(z)$. Then by the uniqueness of the initial value problem (4.3), we have $\tau=\hat{\tau}$. Thus it follows from Lemma 4.1 (3) that $\tau$ is the unique solution of the matrix Ernst equation with the initial value $\left.\tau(z, \rho)\right|_{\rho=0}=\tau(z)$.

Remark 4.4. For any $\tau(z) \in \mathscr{C} \llbracket z \rrbracket$, it follows from Lemma 4.2 that there exists a formal power series

$$
W(z, \rho, \lambda)=1_{2}+\sum_{j=1}^{\infty} w_{j}(z, \rho) \lambda^{-j}, \quad w_{j}(z, \rho) \in \mathscr{C} \llbracket z, \rho \rrbracket
$$

which satisfies conditions (1) and (2) in Theorem 4.3. A solution $\tau(z, \rho)$ of Eqs. (1.2) which satisfies $\left.\tau(z, \rho)\right|_{\rho=0}=\tau(z)$ can be obtained from this $W$ by solving this initial value problem (4.3). Hence every solution of Eqs. (1.2) is constructed in this way with a suitably chosen initial value $\left.\tau(z, \rho)\right|_{\rho=0}=\tau(z)$.

\section{Linearization of the Ernst Equation}

The problem of generating solutions of the matrix Ernst equation has been reduced to find $w_{j}(z, \rho) \in \mathscr{C} \llbracket z, \rho \rrbracket, j \in \mathbf{N}$ such that

$$
\left\{\begin{array}{l}
\rho \partial_{z} w_{j+1}(z, \rho)+\partial_{\rho} w_{j}(z, \rho)=\rho \partial_{z} w_{1}(z, \rho) \cdot w_{j}(z, \rho), \\
\left.w_{j}(z, \rho)\right|_{\rho=0}=w_{j}(z)
\end{array}\right.
$$

where $\tau(z) \cdot[\tau(z+1 / 2 \lambda)]^{-1}=\sum_{j=0}^{\infty} w_{j}(z) \lambda^{-j}$.

Equation (5.1) is easily linearized by using the method of the UGM. Following Takasaki[4] we introduce an $\infty \times \infty$ matrix $\xi=\left(\xi_{i j}\right)_{l \in \mathbf{Z}, l<0}, \xi_{i j} \in \mathscr{C} \llbracket z, \rho \rrbracket$ with the conditions

$$
\begin{gathered}
\Lambda \xi=\xi C, \\
\xi_{i j}=\delta_{i j} 1_{2} \text { for } i, j<0,
\end{gathered}
$$

where $\Lambda=\left(\delta_{i+1, j} 1_{2}\right)_{l, j \in \mathbf{Z}}$ and

$$
C=\left(\begin{array}{c}
\left(\delta_{i+1, j} 1_{2}\right)_{i<-1, j<0} \\
\left(\xi_{0 j}\right)_{j<0}
\end{array}\right) .
$$

Then we have a bijection between $W$ and $\xi$ characterized by

$$
\xi_{0 j}=-w_{-j}, \quad \text { for } \quad j<0 \text {. }
$$


The matrix $\xi$ corresponding to $W$ is represented more explicitly in terms of $w_{j}$ as

$$
\xi=\left(w_{i-j}^{*}\right)_{l \in \mathbf{Z}, j<0} \cdot\left(w_{i-j}\right)_{i, j<0},
$$

where we set $w_{j}=w_{j}^{*}=0$ for $j<0$ and, for $j \geqq 0, w_{j}^{*}$ denotes the coefficients of $W^{-1}$, i.e. $W^{-1}=\sum_{j=0}^{\infty} w_{j}^{*} \lambda^{-j}$.

By using the matrix $\xi$ we rewrite Eqs. (5.1).

Lemma 5.1. Eqs. (5.1) are equivalent to the equations for $\xi$

$$
\rho \partial_{z} \xi_{i+1, j}+\partial_{\rho} \xi_{i j}-\xi_{i,-1} \rho \partial_{z} \xi_{0 j}=0
$$

for $i \in \mathbf{Z}, j<0$.

Proof is completely similar to the derivation of Eqs. (1.14) in Takasaki[4].

A linearization of Eqs. (5.7) is given by the following.

Theorem 5.2. Let $\xi^{(0)}(z)$ be an $\infty \times \infty$ matrix satisfying $(5.2),(5.3)$ and $\xi_{0 j}(z)=$ $-w_{-j}(z), j<0$. Define the $\infty \times \infty$ matrices $\widetilde{\xi}(z, \rho)=\left(\xi_{i j}(z, \rho)\right)_{l \in \mathbf{Z}, j<0}$ and $\widetilde{\xi}_{(-)}(z, \rho)$ by

$$
\begin{aligned}
& \tilde{\xi}(z, \rho)=\exp \left(-\frac{1}{2} \rho^{2} \Lambda \partial_{z}\right) \xi^{(0)}(z)=\sum_{k=0}^{\infty}\left(-\frac{1}{2} \rho^{2} \Lambda \partial_{z}\right)^{k} \xi^{(0)}(z) / k !, \\
& \tilde{\xi}_{(-)}(z, \rho)=\left(\widetilde{\xi}_{i j}(z, \rho)\right)_{i, j<0} .
\end{aligned}
$$

Then the inverse $\tilde{\xi}_{(-)}^{-1}$ and the product $\tilde{\xi}^{-\xi^{-1}}$ can be defined as $\infty \times \infty$ matrices and the matrix

$$
\xi=\tilde{\xi} \cdot \tilde{\xi}_{(-)}^{-1}
$$

satisfies (5.2), (5.3) and (5.7). Furthermore if the initial data $\tau(z)$ is analytic in a neighbourhood of $z=0$, then $w_{j}(z, \rho)=-\xi_{0 j}(z, \rho)$ is analytic in a neighbourhood of $(z, \rho)=(0,0)$.

We can prove this proposition exactly in the same way as Theorem 6 in Takasaki [4].

Remark 5.3. The $\infty \times \infty$ matrix $\tilde{\xi}(z, \rho)$ satisfies the linear differential equation

$$
\partial_{\rho} \tilde{\xi}(z, \rho)=\rho \Lambda \partial_{z} \tilde{\xi}(z, \rho) \text {. }
$$

This equation has already appeared in Nakamura[12] where Ansätz solutions of the Ernst equation are generated.

By using Theorem 5.2 we can construct exact solutions of the Ernst equation.

We consider the case when the initial data $\tau(z)$ is a polynomial of $z$ of degree $m$ such that ${ }^{t} \tau(z)=\tau(z), \operatorname{det} \tau(z)=1$. The value at $\rho=0$ of the wave function is givne by

$$
\left.W(z, \rho, \lambda)\right|_{\rho=0}=\tau(z) \cdot[\tau(z+1 / 2 \lambda)]^{-1}
$$

Since $\tau(z)^{-1}$ is also polynomial of degree $m,\left.W(z, \rho, \lambda)\right|_{\rho=0}$ and $\left.W(z, \rho, \lambda)\right|_{\rho=0}{ }^{-1}$ are polynomials of both $z$ and $\lambda^{-1}$ and have degree $m$ with respect to the variable 
$\lambda^{-1}$. Hence from (5.6) the initial value $\xi^{(0)}(z)=\left(\xi_{i j}^{(0)}(z)\right)_{i \in \mathbf{Z}, j<0}$ is expressed as

$$
\xi(z)=\left(\begin{array}{cc}
\left(\delta_{i j} 1_{2}\right)_{i, j<-m} & 0 \\
0 & \xi(m: z) \\
0 & 0
\end{array}\right)
$$

where $\xi(m: z)=\left(\xi_{i j}(z)\right)_{-m \leqq i \leqq m-1,-m \leqq i<0}$. Then $\tilde{\xi}$ in Theorem 5.2 has the form

$$
\begin{aligned}
& \tilde{\xi}(z, \rho)=\left(\begin{array}{cc}
\left(\delta_{i j} 1_{2}\right)_{l, j<-m} & * \\
0 & \widetilde{\xi}(m: z, \rho) \\
0 & 0
\end{array}\right), \\
& \tilde{\xi}(m: z, \rho)=\left(\widetilde{\xi}_{i j}(z, \rho)\right)_{-m \leqq 1 \leqq m-1,-m \leqq 1<0} .
\end{aligned}
$$

Consequently $\tilde{\xi}(z, \rho)_{(-)}^{-1}$ is

$$
\tilde{\xi}(z, \rho)_{(-)}^{-1}=\left(\begin{array}{cc}
\left(\delta_{i j} 1_{2}\right)_{i, j<-m} & * \\
0 & \xi(m: z, \rho)_{(-1}^{-1}
\end{array}\right) .
$$

Thus we have

$$
\xi(z, \rho)=\left(\begin{array}{cc}
\left(\delta_{i j} l_{2}\right)_{i, j<\cdots m} & 0 \\
0 & \xi(m: z, \rho) \\
0 & 0
\end{array}\right)
$$

where $\xi(m: z, \rho)=\tilde{\xi}(m: z, \rho) \cdot\left(\tilde{\xi}(m: z, \rho)_{(-)}\right)^{-1}$. We remark that $\tilde{\xi}(m: z, \rho)$ and $\tilde{\xi}(m: z, \rho)_{(-)}$ are finite size matrices whose components are polynomials of $z$ and $\rho$. Thus we have the following corollary.

Corollary 5.4. Let $\tau(z) \in \mathscr{C} \llbracket z]$ be a polynomial of $z$ of degree $m$ such that $\operatorname{det} \tau(z)=1$ and ${ }^{t} \tau(z)=\tau(z)$. Then every component of the matrix $\xi$ is a rational function of $z$ and $\rho$.

The unique solutions of the matrix Ernst equation with the initial value $\left.\tau(z, \rho)\right|_{\rho=0}=\tau(z)$ is obtained by solving the initial value problem

$$
\partial_{\rho} \tau=-\hat{c}_{z} \xi_{0,-1} \cdot \tau,\left.\quad \tau(z, \rho)\right|_{\rho=0}=\tau(z) .
$$

We calculate two simple examples:

Examples. Let us consider the initial value problem for the matrix Ernst equation with initial values

$$
\left.\tau(z, \rho)\right|_{\rho=0}=\tau(z)=\left(\begin{array}{cc}
1 & z \\
z & 1+z^{2}
\end{array}\right),\left(\begin{array}{cc}
2 z^{2}+2 z+1 & 2 z^{2} \\
2 z^{2} & 2 z^{2}-2 z+1
\end{array}\right)
$$

respectively. Indeed, both $\tau(z)$ are polynomials of $z$ of degree 2. In these cases we find

$$
\xi_{0,-1}=\left(\begin{array}{cc}
\frac{1}{2}\left(1-\frac{\rho^{2}}{4}\right)^{-1} & 0 \\
z\left(1-\frac{\rho^{2}}{4}\right)^{-1} & -\frac{1}{2}\left(1-\frac{\rho^{2}}{4}\right)^{-1}
\end{array}\right)
$$




$$
\left(\begin{array}{cc}
\frac{1 \rho^{2}-2+4 z^{2}}{2} & -\frac{1 \rho^{2}+4 z+4 z^{2}}{2}-1 \\
\rho^{2}-1 & \rho^{2}-1 \\
\frac{1 \rho^{2}-4 z+4 z^{2}}{2} & -\frac{1 \rho^{2}-2+4 z^{2}}{2} \frac{\rho^{2}-1}{\rho^{2}-1}
\end{array}\right)
$$

and the corresponding solutions of Eqs. (5.8) are

$$
\begin{aligned}
\tau(z, \rho)= & \frac{1}{1-\frac{\rho^{2}}{4}}\left(\begin{array}{c}
1 \\
z
\end{array} \begin{array}{c}
z \\
\left.1-\frac{\rho^{2}}{4}\right)^{2}+z^{2}
\end{array}\right), \\
& \frac{1}{2\left(1-\frac{\left.\rho^{2}\right)}{4}\right.}\left(\begin{array}{cc}
4 z^{2}+4 z+2+\rho^{4}-2 \rho^{2} & 4 z^{2}+\rho^{4}-2 \rho^{2} \\
4 z^{2}+\rho^{4}-2 \rho^{2} & (2 z-1)^{2}+\left(\rho^{2}-1\right)^{2}
\end{array}\right) .
\end{aligned}
$$

The solutions of the Ernst matrix equation are

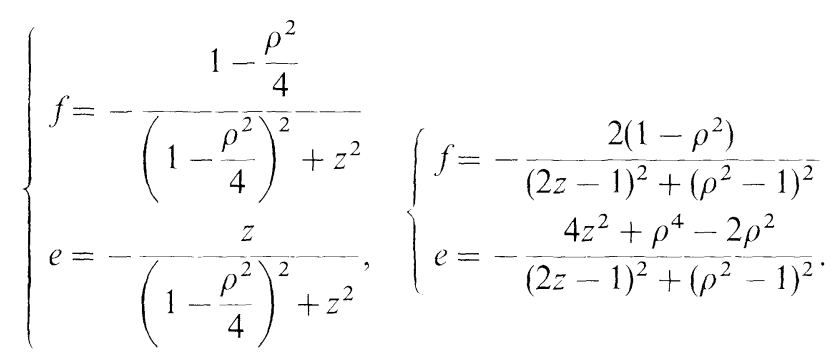

\section{References}

1. Sato, M.: Soliton equations as dynamical systems on an infinite dimensional Grassmann manifold. RIMS Kokyuroku, vol. 439, pp. 30-46. Kyoto University 1981

2. Sato, M., Sato, Y.: Soliton equations as dynamical systems on an infinite dimensional Grassmann manifold. Lecture Notes in Num. Appl. Anal. 5, 259-271 (1982)

3. Mulase, M.: Complete integrability of the Kadomtsev-Petriashv1li equation. Adv. Math. 54, 57-66 (1984)

4. Takasaki, K.: A new approach to the self-dual Yang-Mills equations. Commun. Math. Phys. 94. 34-59 (1984)

5. Suzuki, N.: Witten's gauge field equations and an infinite-dimensional Grassmann manifold. Commun. Math. Phys. 113, 155-172 (1987)

6. Ernst, F. J.: Complex potential formalism to the axially symmetric gravitational fields problem. $J$. Math. Phys. 15, 1409-1412 (1974)

7. Nagatomo, K.: Formal power series solutions of the stationary axisymmetric vacuum Einstein equations. Osaka, J. Math. 25, 49-70 (1988)

8. Hauser, I., Ernst, F. J.: Proof of a Gerouch conjecture. J. Math. Phys. 22, 1151-1163 (1981)

9. Belinsky, V. A., Zakharov, V. E.: Integration of the Einstein equations by means of the inverse scattering problem technique. Sov. Phys. J.E.T.P. 48, $985-994$ (1978); Belinsky, V. A., Zakharov, V. E.: Stationary gravitational solitons with axial symmetry. Sov. Phys. J.E.T.P. 50, 1-9 (1979)

10. Maison, D.: On the complete integrability of the stationary axially symmetric Einstein equation. J. Math. Phys. 20, 871-877 (1979)

11. Nakamura, Y.: Symmetries of statıonary axially symmetric vacuum Einstein equations and the new family of exact solutions. J. Math. Phys. 24, 606 609 (1983)

12. Nakamura, Y.: On a linearization of the stationary axially symmetric Einstein equatıon. Class. Quantum Grav. 4, 437-440 (1987) 
13. Kyriakopoulos, E.: Method of finding axially symmetric stationary vacuum solutions of the equations of general relativity. Phys. Rev. D30, 1158-1160 (1984)

14. Vein, P. R.: Two related families of determinantal solutions of the stationary axially symmetric vacuum Einstein equations. Class. Quantum Grav. 2, 899-908 (1985)

15. Candler, S., Freeman, N. C.: Determinantal solutions of the stationary axially symmetric vacuum Einstein equations. Preprint, University of Newcastel upon Tyne

16. Nagatomo, K.: Explicit description of Ansätze $E_{n}$ for the Ernst equation in general relativity. Preprint, Osaka University

Communicated by H. Araki

Received September 13, 1988; in revised form October 20, 1988 
